\title{
Biomechanical evaluation of the primary stability of three different types of femoral stem fixations for canine total hip replacement
}

Charles Saban ( $\nabla$ charles.saban@vetagro-sup.fr)

VetAgro Sup https://orcid.org/0000-0002-4895-1337

Eric Viguier

VetAgro Sup

Mathieu Taroni

VetAgro Sup

Arnaud Baldinger

VetAgro Sup

Margaux Blondel

VetAgro Sup

Michel Massenzio

Université Claude Bernard Lyon 1: Universite Claude Bernard Lyon 1

Claude Carozzo

VetAgro Sup

Pierre Moissonnier

VetAgro Sup

Thibaut Cachon

VetAgro Sup

Research article

Keywords: Biomechanics, Subsidence, Femoral stem, Dog, Cementless, Cemented

Posted Date: October 8th, 2020

DOI: https://doi.org/10.21203/rs.3.rs-86655/v1

License: (c) (i) This work is licensed under a Creative Commons Attribution 4.0 International License.

Read Full License 


\section{Abstract}

Background: Total hip arthroplasty is the main salvage procedure performed for hip osteoarthritis in dogs. Two main types of femoral stems are available: cemented stems, which offer excellent primary stability but are subject to aseptic loosening in the long term, and cementless stems, which have good long-term outcomes but lack primary stability. The adjunction of a neutral rod through the neck of the cementless stem to the lateral cortex of the femur could offer better primary stability before osteointegration. The aim of this study was to compare the primary stability of three different femoral stems, cemented (cFS), press-fit cementless (pfFS), and rod-press-fit cementless stems (r-pfFS), by measuring their transverse displacement on cyclic assays and resistance to subsidence with unidirectional load compression parallel to the longitudinal axis of the femur.

Results: The force-displacement and stress-strain curves were assessed. The work necessary for subsidence, strain to failure, and mean strain of the cyclic assays were calculated. No significant differences were observed in transverse displacement $(p=0.263)$ or mean strain $(p=0.244)$ during the cyclic tests or in work necessary for subsidence $(p=0.079)$ or strain to failure $(p=0.075)$. The cFS and $r-$ pfFS were significantly more resistant to subsidence than was the pfFS $(p<0,05)$. No significant differences were observed between the cFS and r-pfFS groups $(p=0.48)$.

Conclusions: Cementless femoral stems with transfixing rods offer significantly higher stability to compressive load parallel to the longitudinal axis of the femur than do standard cementless stems and a level of stability comparable to that of cemented stems. r-pfFSs may be valuable in limiting the subsidence and micro-motion of press-fit femoral stems and thus improving the state of osteointegration of the prosthesis during the short-term postoperative period.

\section{Background}

Total hip replacement (THR) is the surgical treatment of choice for hip osteoarthritis in large adult dogs when medical treatment is no longer effective. Hip replacement leads to the total recovery of coxofemoral articulation in 85 to $95 \%$ of cases [1, 2]. Historically, cemented prostheses for THR were the first prostheses released on the veterinary market. The femoral stem is fixed in the bone by adhesion with polymethyl-methacrylate cement (PMMA). After the exothermic reaction and polymerization of the cement, the primary stability is excellent [3]. The short-term clinical outcomes are generally good to excellent in $84 \%$ of cases, according to the owners' perception, with middle- and long-term complication rates ranging from $7-22 \%$ [4-6]. Nevertheless, the complications are often related to the implantation of the bone cement. Indeed, one of the most reported complications in living animals and in post mortem studies is aseptic loosening, and there is a high rate of signs of radiographic changes at eight weeks postoperatively or at necropsy, affecting $63,7 \%$ of the dogs studied $[4,7,8]$. Infection is also a concern because cement acts as a foreign body in the femoral shaft [5]. 
To avoid these limitations, cementless femoral stems have been developed. In these kinds of prostheses, primary stability is achieved by press-fit fixation and is solely based on the force of friction. The stem is covered by a porous material composed of titanium or hydroxyapatite, which permits osteointegration during bone healing and provides excellent long-term stability with a return to normal articular function in 80 to $88 \%$ of cases $[9,10]$. Cementless femoral stems have several advantages: they decrease the surgical time, limit infections, and decrease the risk of long-term implant loosening [10]. However, during the osteointegration period, only forces of friction with small surfaces of osseous contact maintain the system; thus, subsidence is possible. Indeed, subsidence has been commonly reported as a short-term complication in clinical studies [10].

To overcome the major complications of each type of implant (aseptic loosening for cemented stems and the lack of primary stability for cementless stems), a new type of implant has been designed. On a standard cementless femoral stem, a hole was added in the long axis of the neck of the stem, permitting the adjunction of a transfixing rod that passes through the prosthesis to the lateral aspect of the femur and distal to the greater trochanter. It was hypothesized that this rod-press-fit cementless stem (r-pfFS) stabilizes and reinforces the cementless system, limiting the torsion and compression forces during osseointegration and bone healing.

The aim of this study was to biomechanically validate this new implant by comparing its primary stability to that of the two other types of femoral stems, cemented (cFS) and press-fit cementless stems (pfFS), after cyclic assays and load compression to failure with a unidirectional servohydraulic press.

\section{Results}

The pre-implantation digital radiographs showed normal conformation of the femurs without growth plates. The average diameter of the proximal femoral shaft was $19.4 \pm 1.4 \mathrm{~mm}$, which was sufficient for the implantation of standard-sized femoral stems (7.5). During implantation, no fractures were created during the impaction of the cementless stems or during the positioning of the transfixing rod. Each rod was placed in the target area, distal to the greater trochanter in the femoral lateral cortex, without modifications of the stem's anteversion angle. On the post-implantation radiographs, no fractures were identified in the groups, and PMMA cement was homogeneously distributed around the stem in the femoral shaft. The frontal (varus-valgus) and sagittal (cranio-caudal) angles were $2.22 \pm 0.76^{\circ}$ and $3.02 \pm$ $1.44^{\circ}$, respectively. No significant differences were observed between the groups $(p=0.65$ and 0.81 , respectively).

In the cyclic assays, there was no significant difference between the groups in transverse displacement $(p=0.26)$ or mean strain $(p=0.24)$.

In the failure tests, no significant differences were observed in strain to failure $(p=0.075)$. For the cFSs and r-pfFSs, the work necessary for subsidence was similar, but no significant differences were observed between these stems and the pf-FSs. Significant differences were observed between the cFS-pfFS $(p=$ 
$0.025)$ and r-pfFS-pfFS ( $p=0.041)$ groups in load to failure. However, no significant differences were observed between the cFS and r-pfFS groups $(p=0.48)$ (Fig. 1).

In every group, all the fractures that occurred after the failure tests were long oblique fractures. In the cFS and r-pfFS groups, the fractures were located on the cranial aspect of the femur along the medio-distal direction, whereas in the pfFS group, the fractures were located on the medial aspect of the femur. The origin of the fractures was located at the level of the press-fit femoral stem collar in the caudo-lateral direction (Fig. 2).

\section{Discussion}

Before a clinical study in living patients is conducted, biomechanical studies need to be conducted to confirm the feasibility of the surgical technique and to test the effectiveness of the system in laboratory conditions. The majority of the parameters reviewed in this biomechanical study showed no significant differences between study groups. However, the load to failure was significantly higher for the new implant (r-pfFS) and cFS than for the standard press-fit femoral stem (pfFS). Cementless femoral stems with a transfixing rod offer significantly higher stability than do standard cementless stems, without significant differences between the stems in transverse displacement. r-pfFSs may be valuable, as they limit the compression and torsional forces and promote bone healing.

Cemented stems have better primary stability, with values that were 2 and 1.5 times higher than those for pfFSs and r-fFSs, respectively, and the difference between the cFS and pfFS groups was significant [5]. Studying the "interface" is fundamental to understanding how femoral stems remain bonded to the femoral shaft. When materials with different properties (bone, cement, implant) come in contact, an interface is created. The load of the implant is therefore distributed to the surrounding bone along the bone-implant interface. Material properties are defined by stiffness, shape, and surface architecture. The load transitions between materials created by the interface need to bear large amounts of stress, and their ability to near this stress is defined by the stiffness ratio of each material [11]. On the cemented stem, three interfaces are present: the cement-implant interface, inner cement mantle, and cement-bone interface. These interfaces permits the distribution of load on the different elements. Moreover, excellent primary stability can be possible by the cohesive action of the PMMA, which acts as grout. Bone cement penetrates the micro-irregular grooves of the reamed bones and is responsible for the shear forces at the interface. However, cement acts as a foreign body: polypropylene debris can migrate into the cancellous bone, enhancing the pro-inflammatory response and thus improving the risk of aseptic loosening [12].

In press-fit femoral stems, the initial fixation is obtained by press-fitting an oversized femoral stem in the femoral shaft to create primary stability with only frictional forces. Moreover, the stiffness of the cementless implant being higher than that of the bone leads to overloading of the implant and thus stress shielding of the bone. The results obtained in this study are in agreement with those in the literature $[13,14]$. Work necessary for subsidence was lower for the pfFS than for the cFS and r-pfFS, although the differences were not significant. Indeed, although frictional forces can bear initial loading, 
load to failure and thus the work necessary for subsidence was significantly lower for the pfFS. This difference may be related to the lack of secondary fixation, such as fixation with cement, which acts as grout for the cemented stem and the rod for the r-pfFS.

The addition of a transfixing rod in neutralization in the neck of a cementless press-fit femoral stem allows the resistance to subsidence to be significantly higher than that of the standard cementless stem and the stability after cyclic loading to be similar to that of the cemented femoral stem. Bucks et al. compared the resistance of subsidence in a standard press-fit femoral stem and an interlocking femoral stem, and the peak load to failure was always significantly higher for the interlocking stem than for the standard press-fit femoral stem: $2.337 \pm 782 \mathrm{~N}$ and $1.405 \pm 712 \mathrm{~N}$, respectively [13]. The resistance to subsidence was greater with a transfixing neutral rod on the press-fit cementless femoral stem than with the standard femoral stem. Clinically, the results are consistent with those of the study by Mitchell et al., in which different types of cementless stems were analysed [15]. Although the systems are not the same but are biomechanically similar, the lateral bolt femoral stem was associated with less subsidence in the postoperative period than was the standard stem. The rod strengthens the system by limiting the compression and torsional forces during axial loading, significantly increasing the solidity of the system without the need for additional complex surgical procedures. These results are encouraging; the r-pfFS system is similar to that of the cFS in terms of primary stability, with a similar magnitude of work necessary for subsidence. Moreover, the rod could be easily and quickly placed in the lateral cortex in the single hole under direct guidance by the prosthetic femoral neck, without inducing fracture or altering the stem's anteversion angle, emphasizing the feasibility of the surgical technique. The procedure appears to be less complicated than that for other hybrid implants, such as interlocking femoral stems. However, the simple adjunction of the rod could lead to less stability and an increased risk of rod migration than do the other systems $[13,14]$. Furthermore, there are no data on how well the rod stays in place in the long term. Indeed, whether the micromotion of the stem during gait can move or break the rod, increasing its potential for migration, has yet to be determined. It is nevertheless essential to perform the drilling procedure with care to prevent damage to the sciatic nerve, which is close to the surgical site [16].

Proper positioning of the femoral stem is a critical aspect during surgery as well as during biomechanical assays and is strongly related to the surgeon's skills at the moment of positioning and impaction of the stem. In this study, all stems were implanted by a single experienced, board-certified surgeon. All the stems were well positioned with varus-valgus and craniolateral angles in concordance with those reported in the literature, and there were no significant differences between groups. This parameter needs to be assessed before any assays are performed. Indeed, if only subjective eye evaluations are performed after implantation, slight differences in angulation can affect the final result. It has been shown that varus angle of the femoral stem greater than or equal to $5^{\circ}$ leads to an increased risk of fracture intraoperatively because of the medial position of the proximal part of the femoral stem, which overpressures a common site of fractures, the craniomedial part of the proximal femur [17].

The neutral position of the femoral stem permits an ideal distribution of the strain. The hole drilled in the trochanteric fossa maximizes the chance of adequate angulation of the stem in the femoral shaft. After 
head and neck ostectomy, the proximal part of the femur has a typical " 8 " shape formed by the intact greater trochanter and the distal part of the neck. The centre of the " 8 " shape corresponds to the trochanteric fossa and is in the same plane as the anatomic axis of the femur for optimal placement of the stem. If a hole is reamed on the lower circle of the " 8 " shape, the hole will naturally exhibit varus angulation, following the direction of the femoral neck, which can lead to incorrect positioning of the stem. The enlargement of the trochanteric fossa therefore provides good angulation of the stems. For press-fit prostheses, the position of the stem can have low precision; indeed, placing the stem with a hammer induces high variability in the stem position at the moment of impaction.

All the fractures in the groups were long, oblique fractures and were similar to fractures generally observed to lead to natural complications [18]. However, the location of the fractures differed between groups. In the pfFS group, the fractures were on the medial aspect of the femur, with its origin on the craniomedial part of the proximal femur. This location represents the most common site of fractures, and it is generally due to the varization of the femoral stem $[17,19]$. The absence of a uniform force distribution might cause fractures in this area. During failure tests, the stem might bend in relation to the femoral shaft, increasing its varus angle and reaching a critical position until failure. Unfortunately, no digital images were available to measure the displacement during the failure tests. In the cFS and r-pfFS groups, the fractures were located on the cranial aspect of the femur. The good neutral position confirmed by the post-implantation radiographs suggests that the forces were equally distributed between the two groups and emphasizes the advantages of the neutral rod in r-pfFSs over pfFSs.

Cyclic assays have been developed with the technical capabilities of a servo-hydraulic press. The aim of the test was not to imitate the immediate postoperative normal gait of the dog after surgery but rather to pre-stress the femur before the failure test. Immediately postoperatively, dogs walk for approximately 1500 steps [20]. For the assays presented in this study, only 90 cycles were performed. Considering the relatively low number of cycles, the load was set to be $75 \%$ of the dog's living body weight, and the trot was mimed to increase the stress on the femur [21]. No significant difference was observed between all groups. Moreover, the p-value corresponding to the difference between the cFS and r-pfFS groups was highly non-significant, indicating a similar magnitude of transverse displacement. These results validate the reliability of the new implant and demonstrate that the new implant yields the same strain and displacement as the other groups. The results are concordant with those in previous studies, with values of $0.70 \pm 1.21 \mathrm{~mm}$ and $0.35 \pm 0.41 \mathrm{~mm}$ for the standard press-fit femoral stem and with the interlocking nail femoral stem, respectively [13].

However, this study has several limitations. The standard size of the femoral stems used in this study can be a limitation. However, it appears that the canals filled by stems are poor indicators for identifying good-sized stems and carry poor clinical relevance [17]. The relatively low number of femurs studied can influence our statistical results. Moreover, the low number of cycles achieved in the assays may not precisely characterize the immediate postoperative period in living dogs. As a biomechanical study is always a simplification of what truly occurs in nature, the femurs were not subject to physiologic forces encountered during normal canine gait. Indeed, the actions of the gluteal and adductor muscles and the 
resulting rotational and shear forces were not taken into account during the assays. Moreover, the axis of the femur during the compression tests did not correspond to the physiological axis of the femur during weight-bearing in the animal's daily life.

\section{Conclusions}

In this study, a new femoral implant for total hip arthroplasty was biomechanically characterized in comparison to a pre-existing femoral stem. The addition of a transfixing neutral rod improves the primary stability of a cementless femoral stem to a level similar to that of the cemented stem. Additional studies, especially in vivo, are mandatory to evaluate the results of the implantation of a neutral femoral stem in the short, mid, and long term.

\section{Methods}

\section{Femur preparation}

Nine pairs of femurs were harvested from experimental adult dogs ( $23.45 \pm 5.22 \mathrm{~kg}$; mean $\pm S D)$, humanely euthanized with medetomidine $30 \mu \mathrm{g} / \mathrm{kg}$ IV (Domitor, Orion Corporation, Finland), Butorphanol 0,3 mg/kg IV (Dolorex, Zoetis, France) and Pentobarbital 182,2 mg/kg IV (Dolethal, Vetoquinol, France) for purposes unrelated to the study. The local ethical committee approved the study. The ages of the dogs were unknown, and sex varied. The muscles and tendons were detached from the bones. Mediolateral and craniocaudal radiographs of the bone samples included in the study were taken to measure the femoral length and the proximal femoral diameter and validate the standard stem size (7.5) used in this work. The bones were then wrapped in towels soaked with $0.9 \% \mathrm{NaCl}$ solution, placed in congelation bags, and stored at $-20^{\circ} \mathrm{C}$ until implantation. The femurs were randomly divided into three groups by the type of femoral stem using a Latin square design; care was taken so that the same femur of a single pair was not included in the same group.

Three different types of femoral stems were used in this study, and all the stems were supplied by the same manufacturer (PorteVet, Porticcio, France). The cemented stem (cFS); the press-fit cementless stem (pfFS), coated with titanium on the proximal part of the stem; and the rod-press fit cementless stem ( $r$ pfFS), which had the same design as the pfFS with the adjunction of a hole in the neck of the stem so that a rod could pass through the prosthesis to the lateral aspect of the femur distally toward the greater trochanter.

The femurs were defrosted 12 hours before implantation at room temperature. The protocols of implantation were almost the same for all stems and met the manufacturer's recommendations. All femoral stems were implanted by a single board-certified surgeon (TC). For the cFS, ostectomy of the head and neck was performed with a $10 \mathrm{~mm}$ oscillating saw (Colibri II DePuy Synthes, Oberdoff, Switzerland) using a specific lateralized guide that passed by the greater trochanter and moved proximally toward the lesser trochanter. A $3 \mathrm{~mm}$ hole was drilled parallel to the anatomic axis of the 
femur in the trochanteric fossa, and the hole was enlarged with a bone rongeur. Gradually, the femoral shaft was power-reamed with bore pliers of a different diameter until it fit the standard size of the stem (7.5). A cement restrictor plug was placed in the bottom of the drilled shaft, and the femoral stem was fixed with radio-opaque bone cement (Bone cement PMMA DePuy CMW3 medium viscosity, Blackpool, Lancashire, UK); care was taken so that the anteversion angle did not change during polymerization. For the pfFS, the same protocol was used, and the hole was not too wide to provide good impaction of the femoral stem. The pfFS was placed in the femoral shaft with a specific hammer for impaction. Particular attention was paid to prevent iatrogenic fractures of the proximal part of the femur during implantation. The r-pfFS had a $2.1 \mathrm{~mm}$ hole in the femoral neck where the rod was placed. In the same manner, after standard implantation of the r-pfFS, a $2 \mathrm{~mm}$ rod was placed from the femoral neck to the lateral aspect of the femur. After the femoral stem was placed in a good position, the rod naturally moved in the lateral cortex, just distal to the greater trochanter. The rod was then cut for the two extremities, and it was positioned appropriately in the femoral head.

After implantation, cranio-caudal and mediolateral digital radiographs were taken (Fig. 3). To ensure the reliable positioning of the femoral stem and repeatability of the biomechanical assays, the varus-valgus and cranio-lateral angles were calculated [17]. On the cranio-caudal and mediolateral radiographs, the angle between the long axis of the stem and proximal anatomical axis of the femurs was recorded to calculate the varus-valgus and cranio-caudal angles, respectively (OSX Horos v3.3.5) (Fig. 4). The femoral heads were then placed on each femoral stem with a head impactor.

\section{Biomechanical assays}

The distal parts of femurs were potted in a standard-sized PVC tube $(60 \mathrm{~mm} * 40 \mathrm{~mm})$ with synthetic polyurethane polymeric resin (Resine Axson, Distrib. ETS Vaillat SAS Oyonnax France). Then, two dots were drawn in the middle of the cranial and lateral aspects of the bone and potted using a vertical laseroptic measuring tool (Laser Level Bosch Quigo, Gerlingen, Germany) that joined the dots to ensure proper positioning of the femurs, with the proximal anatomical axes positioned to be vertical to the ground (Fig. 5). After polymerization, the tubes were cut and removed.

A unidirectional servohydraulic press was used for this study (Shimadzu AGS X-series, Kyoto, Japan). The "femur-resin" systems were then distally fixed to a specially designed base and proximally to a manufactured cup for the femoral head. The systems were placed so that the direction of the compression force was parallel to the longitudinal axis of the femur (Fig. 6).

The femurs were first subjected to cyclic assays. The system was initially preloaded at $10 \mathrm{~N}$. Then, ninety cycles of axial compression were performed at $75 \%$ of the living body weight of the dogs $(182 \pm 36.4 \mathrm{~N})$ and $0.2 \mathrm{~mm} / \mathrm{s}$ to assess the strain and transverse displacement. A cycle was defined from the moment compression started to the moment of peak load and pre-load charge were reached (Fig. 7).

After the cyclic assays, the femurs were loaded to subsidence with a unidirectional load at $0.2 \mathrm{~mm} / \mathrm{s}$. The data were recorded at $100 \mathrm{~Hz}$ using the manufacturer's software (Shimadzu Trapezium X, Kyoto, Japan) 
for both the cyclic and resistance to subsidence assays. After the failure tests, the type and localization of the fractures were recorded with digital photographs.

\section{Data Analysis}

For the failure tests, the force recorded corresponded to the magnitude of displacement during the assay. A force-displacement curve was then plotted for each femur. The force to subsidence, displacement to subsidence and work necessary for subsidence were calculated. The work necessary for subsidence represented the area under the force-displacement curve (Microsoft Excel OSX v16.32, Redmond, USA). For the failure and cyclic tests, the strain was calculated using the displacement during the assays and the length of each implanted femur to the most proximal point of the femoral stem's neck to the most distal point of the femur.

All data are expressed by descriptive statistics, and non-parametric Kruskal-Wallis tests were used to statistically compare the differences between the groups. Nonparametric Wilcoxon tests were used to compare the differences between paired groups. The confidence interval was set to be 0.05 (R 3.5.2 GUI 1.70 El Capitan build, Vienna, Austria).

\section{List Of Abbreviations}

cFS: cemented femoral stem

pfFS: standard press-fit cementless femoral stem

r-pfFS: transfixing-rod press-fit cementless femoral stem

\section{Declarations}

\section{Ethics approval and consent to participate}

Not applicable

\section{Consent for publication}

Not applicable

\section{Availability of data and materials}

The datasets generated and/or analysed during the current study are available from the corresponding author upon reasonable request.

\section{Competing interests}

The authors declare that they have no competing interests. 


\section{Funding}

The femoral stems, specific bases and manufactured cups used for the biomechanical study were provided by PorteVet Society.

\section{Author contributions}

CS: Study design, data acquisition and analysis, statistical analysis, manuscript drafting. TC: Study design, data acquisition, surgical procedures, review and revision of the manuscript. EV: Study design and review and revision of the manuscript. $M T, A B, M B, P M$, and $C C$ : Review and revision of the manuscript. All authors have read and approved the final manuscript and have agreed to be accountable for all aspects of the work.

\section{Acknowledgements}

The authors would like to thank Mr. Michel Porte and PorteVet ${ }^{\circledR}$ society for providing the femoral stems. They would also like to thank the laboratory of biomechanics of the ICE research unit (Interactions Cells Environment).

\section{References}

1. Olmstead ML. Total hip replacement. Vet Clin North Am Small Anim Pract. 1987 Jul;17(4):943-55.

2. Paul HA, Bargar WL. Histologic changes in the dog acetabulum following total hip replacement with current cementing techniques. The Journal of Arthroplasty. 1987 Jan;2(1):71-6.

3. Siopack JS, Jergesen HE. Total hip arthroplasty. West J Med. 1995 Mar;162(3):243-9.

4. Skurla CT, Egger EL, Schwarz PD, James SP. Owner assessment of the outcome of total hip arthroplasty in dogs. Journal of the American Veterinary Medical Association. 2000 Oct;217(7):1010-2.

5. Olmstead. Canine cemented total hip replacements: Sthe of the art. JSAP. 1995;36:395-9.

6. Edwards MR, Egger EL, Schwarz PD. Aseptic loosening of the femoral implant after cemented total hip arthroplasty in dogs: 11 cases in 10 dogs (1991-1995). J Am Vet Med Assoc. 1997 Sep 1;211(5):580-6.

7. Bergh MS, Gilley RS, Shofer FS, Kapatkin AS. Complications and radiographic findings following cemented total hip replacement: A retrospective evaluation of 97 dogs. Veterinary and Comparative Orthopaedics and Traumatology. 2006;19(03):172-9.

8. Bergh MS, Muir P, Markel MD, Manley PA. Femoral Bone Adaptation to Unstable Long-Term Cemented Total Hip Arthroplasty in Dogs. Veterinary Surgery. 2004 May;33(3):238-45.

9. Vezzoni L, Vezzoni A, Boudrieau RJ. Long-Term Outcome of Zürich Cementless Total Hip Arthroplasty in 439 Cases: Long-Term Outcome of Zürich Total Hip Arthroplasty. Veterinary Surgery. 2015 Nov;44(8):921-9. 
10. Kidd S, Preston C, Moore G. Complications of porous-coated press-fit cementless total hip replacement in dogs. Vet Comp Orthop Traumatol. 2016 Sep;29(05):402-8.

11. Kowaleski MP. Biomechanical Considerations in Total Hip Replacement. In: Peck JN, Marcellin-Little DJ, editors. Advances in Small Animal Total Joint Replacement [Internet]. West Sussex, UK: John Wiley \& Sons, Inc.; 2013 [cited 2019 Sep 13]. p. 53-68. Available from: http://doi.wiley.com/10.1002/9781118704776.ch6

12. Massin P, Viguier E, Flautre B, Hardouin P, Astoin E, Duponchel B. Migration of polyethylene debris along well-fixed cemented implants. J Biomed Mater Res. 2004 Feb 15;68B(2):140-8.

13. Buks Y, Wendelburg KL, Stover SM, Garcia-Nolen TC. The Effects of Interlocking a Universal Hip Cementless Stem on Implant Subsidence and Mechanical Properties of Cadaveric Canine Femora: BFX ${ }^{\circledR}$ Stem Modification Using an Interlocking Bolt. Veterinary Surgery. 2016 Feb;45(2):155-64.

14. Ordway NR, Ash KJ, Miller MA, Mann KA, Hayashi K. A Biomechanical Comparison of Four Hip Arthroplasty Designs in a Canine Model. Vet Comp Orthop Traumatol. 2019 Sep;32(05):369-75.

15. Mitchell MM, Hudson CC, Beale BS. Comparison of femoral stem subsidence between three types of press-fit cementless total hip replacement in dogs. Veterinary Surgery. 2020 Feb 21;vsu.13391.

16. Andrews CM, Liska WD, Roberts DJ. Sciatic Neurapraxia As a Complication in 1000 Consecutive Canine Total Hip Replacements. Vet Surgery. 2008 Apr;37(3):254-62.

17. Townsend S, Kim SE, Pozzi A. Effect of stem sizing and position on short-term complications with canine press fit cementless total hip arthroplasty. Veterinary Surgery. 2017 Aug;46(6):803-11.

18. Ganz SM, Jackson J, VanEnkevort B. Risk Factors for Femoral Fracture after Canine Press-Fit Cementless Total Hip Arthroplasty: Press-Fit Cementless Total Hip Arthroplasty. Veterinary Surgery. 2010 Apr;no-no.

19. Pernell RT, Gross RS, Milton JL, Montgomery RD, Wenzel JGW, Savory CG, et al. Femoral Strain Distribution and Subsidence After Physiological Loading of a Cementless Canine Femoral Prosthesis: The Effects of Implant Orientation, Canal Fill, and Implant Fit. Veterinary Surgery. 1994 Nov;23(6):503-18.

20. Aper RL, Litsky AS, Roe SC, Johnson KA. Effect of bone diameter and eccentric loading on fatigue life of cortical screws used with interlocking nails. Am J Vet Res. 2003 May;64(5):569-73.

21. Budsberg SC, Verstraete MC, Soutas-Little RW. Force plate analysis of the walking gait in healthy dogs. Am J Vet Res. 1987 Jun;48(6):915-8.

\section{Figures}



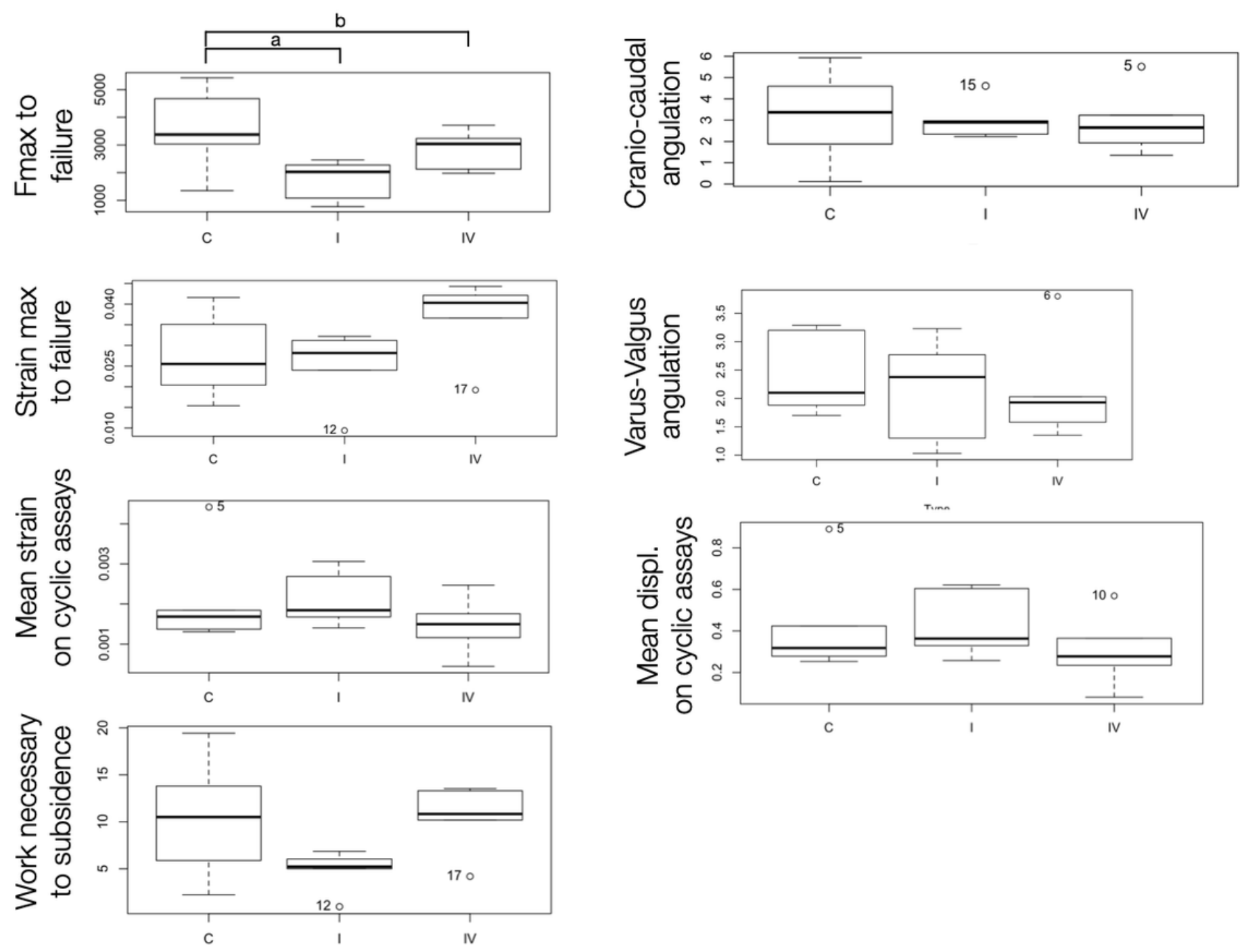

Figure 1

Box plots summarizing the results of the cyclic and subsidence assays. Significant differences are indicated by connected lines. "displ." means displacement. 

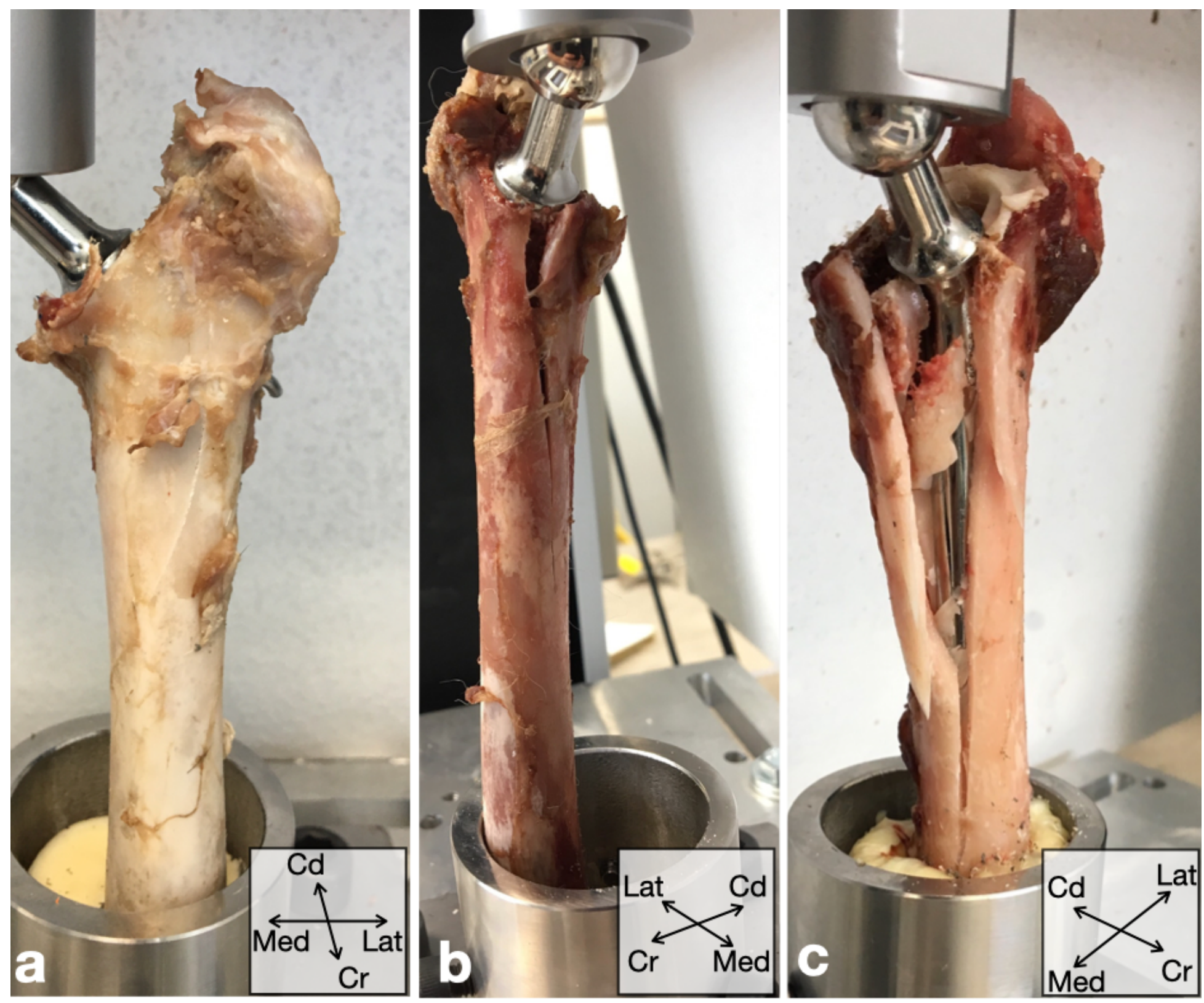

Figure 2

Localization of the fractures with the a) r-pfFS, b) pfFS, and c) cFS. a) and c) The origin of the fracture is on the cranial aspect of the femur in the medio-distal direction. b) The origin of the fracture is on the medial aspect of the femur at the level of the stem collar. 


$$
\text { [II] }
$$




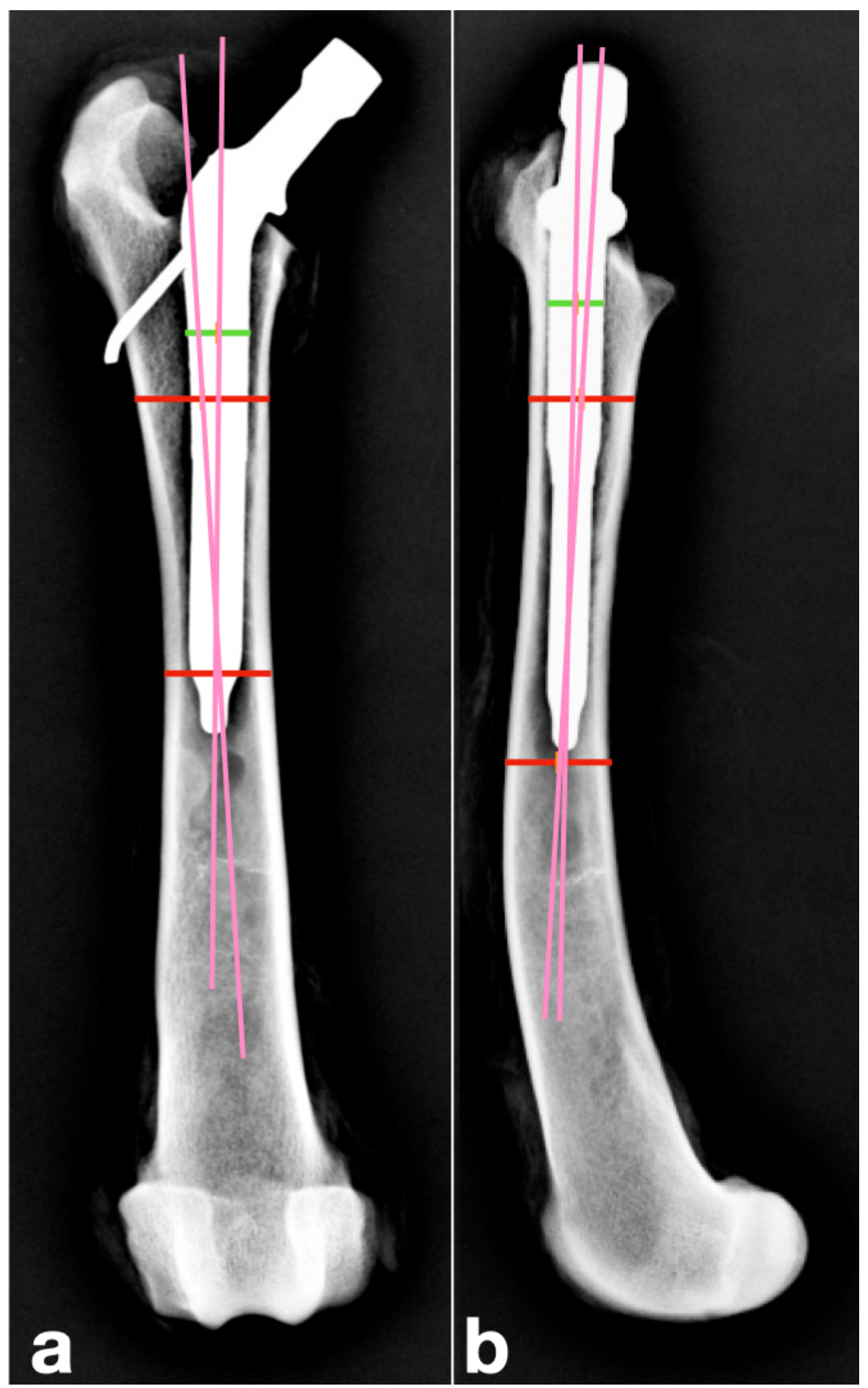

\section{Figure 4}

Varus-valgus and cranio-caudal angles of the stem shown on the cranio-caudal and medio-lateral radiographs, respectively. The red lines represent the proximal diameter of the femoral shaft, and the green line represents the proximal diameter of the femoral stem. The angle formed by the pink line passing through the middle of the red lines and the angle formed by the caudal part and middle of the green line represent the varus-valgus and cranio-caudal angles, respectively. 


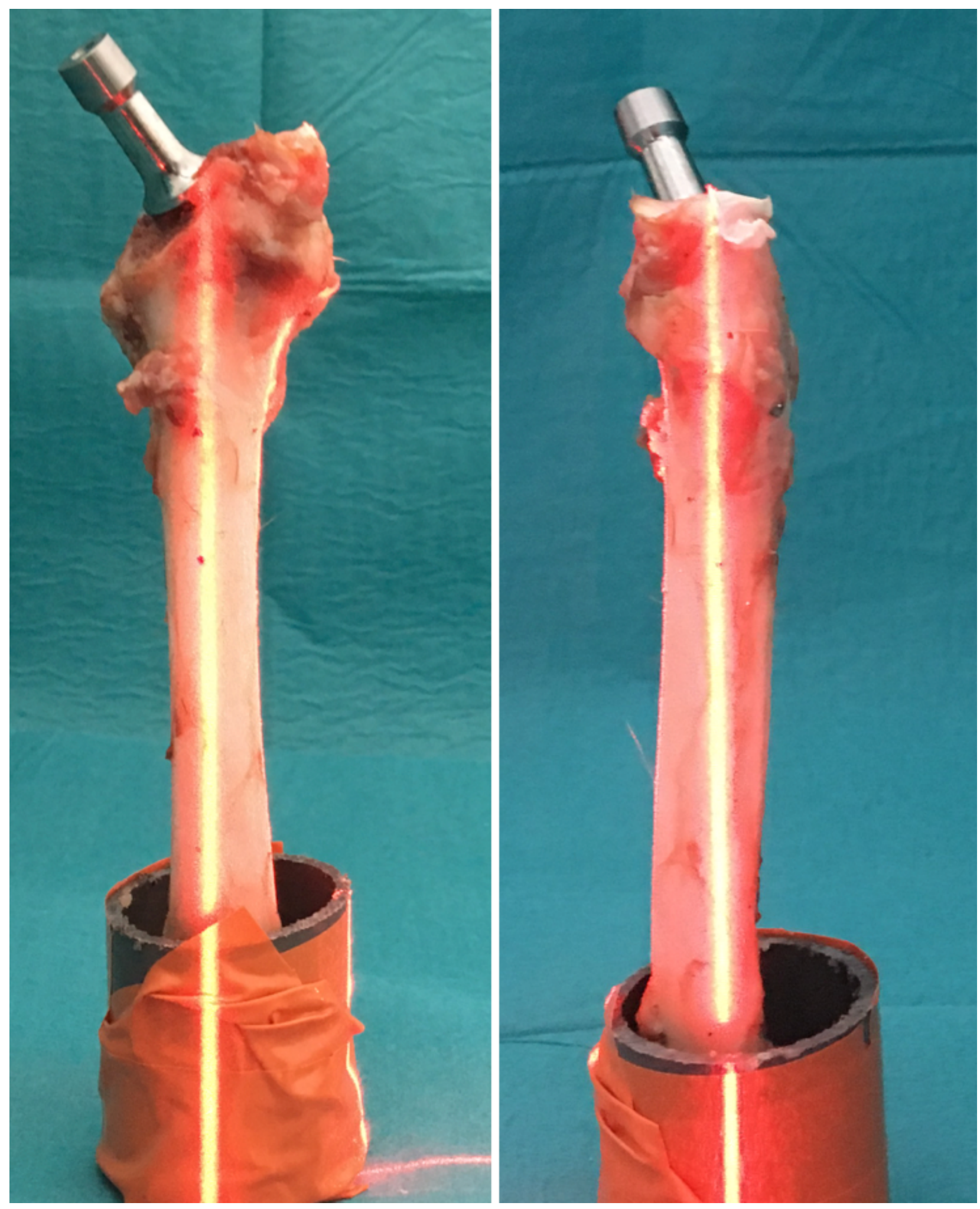

\section{Figure 5}

Positioning of the implanted femurs in the resin. a) Cranio-caudal view; b) latero-medial view. Note that the two dots represent the centre of the proximal shaft of the femur, where the laser light passes through. 


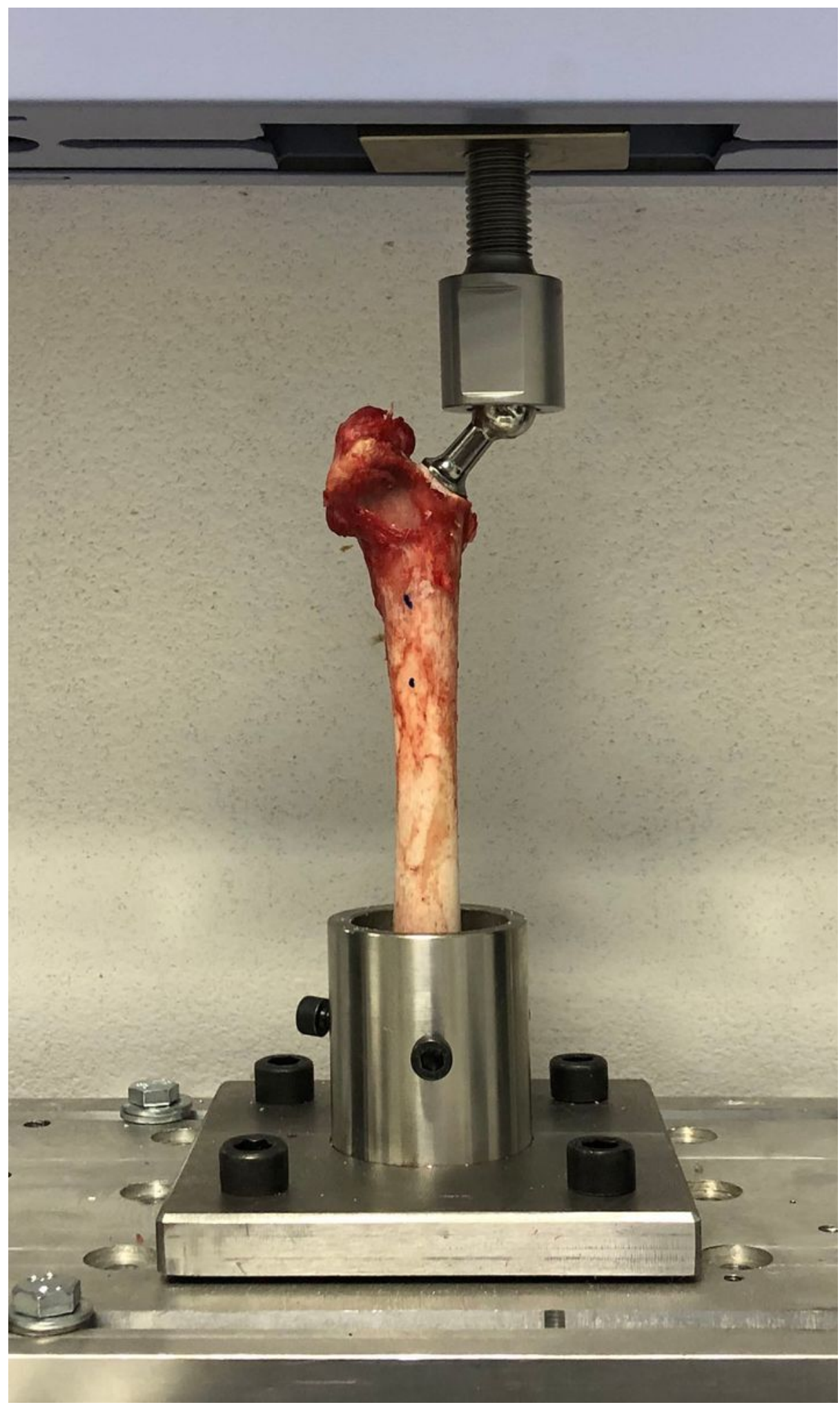

Figure 6

The "femur - femoral stem" system in the servohydraulic press before the biomechanical assays were performed. 
Cyclic Assay of a cFS

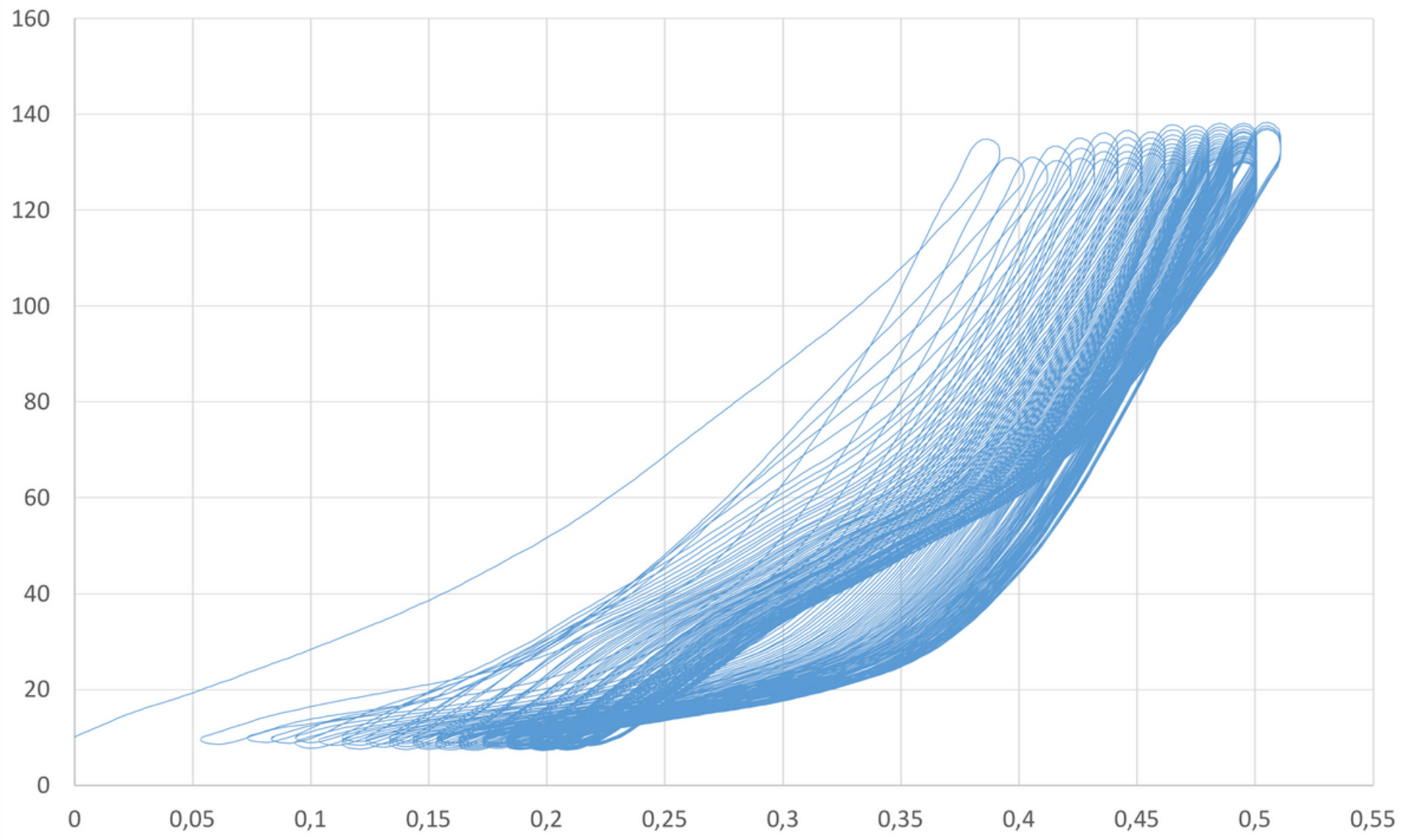

\section{Figure 7}

Example of the hysteresis curve plotted during the cyclic assays for a stem in the cFS group. The $x$-axis is in millimetres $(\mathrm{mm})$, and the $y$-axis is in Newtons $(\mathrm{N})$. One loading/unloading cycle corresponds to a cycle in the curve. 